\title{
Effect of Rapid Evolution of Magnetic Tilt Angle of a Newborn Magnetar on Light Curve of Gamma-ray Burst
}

Ming $\mathbf{X u}^{*+}$

Department of Physics, Jiangxi Science \& Technology Normal University, Nanchang 330013, China

E-mail: xumingenju.edu.cn

\begin{abstract}
We study the electromagnetic radiation from a newborn magnetar whose magnetic tilt angle decreases rapidly and its effect on light curve of gamma-ray bursts (GRBs). We calculate the evolution of the angular spin frequency, the perpendicular component of the surface magnetic field strength, and the energy loss rate through magnetic dipole radiation. We show that the spin-down of the magnetar experiences two stages characterized by two different timescales. The apparent magnetic field decreases rapidly with the decrease of the tilt angle. It is shown that the evolution of the energy loss rate is consistent with the overall light curves of those gamma-ray bursts which show a plateau structure in the afterglow stage, such as GRB 061121 and GRB 090426 etc. The energy loss rate of the magnetar with rapid evolution of magnetic tilt angle is very different from that of a fixed tilt angle case. Our model supports the idea that the GRBs with a plateau phase in afterglow may be originated from newborn millisecond magnetars.
\end{abstract}

Swift: 10 Years of Discovery,

2-5 December 2014

La Sapienza University, Rome, Italy

\footnotetext{
* Speaker.

${ }^{\dagger}$ This work was supported by the National Natural Science Foundation of China (Grant No. 11203020).
} 


\section{Introduction}

A newborn magnetar with a millisecond rotational period could provide enough rotational energy to power the GRB ejecta [W]. Along with the the magnetar slowly spins down, the rotational energy released will provide a continuous energy-injection to the GRB jet and produce a plateaulike structure in the afterglow light curve [2].

The dipole magnetic fields of magnetars are required to be higher than $10^{15} \mathrm{G}$ since a significant portion of the rotational energy should be released on the timescale of the GRB duration. On the other hand, the magnetars should have a dipole magnetic field less than $10^{15} \mathrm{G}$ for explaining the relatively long-lasting shallow decays in the afterglow light curves of GRBs [B]].

We suggest that the contradiction of the magnetic field requirements could be solved by considering the evolution of the magnetic tilt angle of the magnetar. Due to the rapid decrease of the tilt angle, the perpendicular component of the magnetic dipole field at the surface of the magnetar is also decreasing rapidly. As a result, the apparent magnetic field can be very high in the beginning, but will be much lower at later times. Our scenario can well explain the overall light curves of those GRBs that have a plateau structure in the afterglow stage.

\section{Numerical calculations and results}

Through magnetic dipole radiation [四], the newborn magnetar loses energy at a rate of

$$
\dot{E}=-\frac{2}{3 c^{3}}|\ddot{m}|^{2}
$$

where $c$ is the speed of light in vacuum, $m$ is the dipolar moment of the magnetic field. We assume that the magnetar rotates at an angular frequency of $\Omega$, and that the magnetic dipole moment $m$ orients at an angle $\alpha$ to the rotation axis.

We assume that the tilt angle $\alpha$ is decaying evenly with time as

$$
\alpha=\alpha_{0}-K \cdot t
$$

where $\alpha_{0}$ is the initial tilt angle and $K$ is a constant characterizing the decreasing speed [ $[$ ].

On the other hand, the radiation energy originates from the rotational kinetic energy of the magnetar, $E=\frac{1}{2} I \Omega^{2}$, where $I$ is the moment of inertia of the magnetar. Thus the energy loss rate

$$
\dot{E}=I \Omega \dot{\Omega} .
$$

Due to the loss of the rotational energy, the magnetar slows down with time.

With the following typical initial parameters [[]], $B_{\mathrm{p}}=1.0 \times 10^{16} \mathrm{G}, R=1.2 \times 10^{6} \mathrm{~cm}, I=$ $2.0 \times 10^{45} \mathrm{~g} \cdot \mathrm{cm}^{2}, P_{0}=1.0 \times 10^{-3} \mathrm{~s}, \alpha_{0}=\pi / 3 \mathrm{rad}, \alpha_{\text {end }}=0.06$ and $t_{\text {end }}=100.0 \mathrm{~s}$, we calculate the aforementioned differential equations.

As is shown in Fig. 囬, the evolution of the angular frequency of a magnetar includes two stages with the decrease of the tilt angle [ $[$ ] $]$. For comparison, we also show the spin-down evolution of magnetars without tilt angle evolution in Fig. $\mathbb{U}$.

The evolution of the energy loss rate of the magnetar can also be divided into two stages [5]. As shown in Fig. $\square$, at first, $\dot{E}$ decays along the dashed curve, similar to the evolution of $\dot{E}$ for 


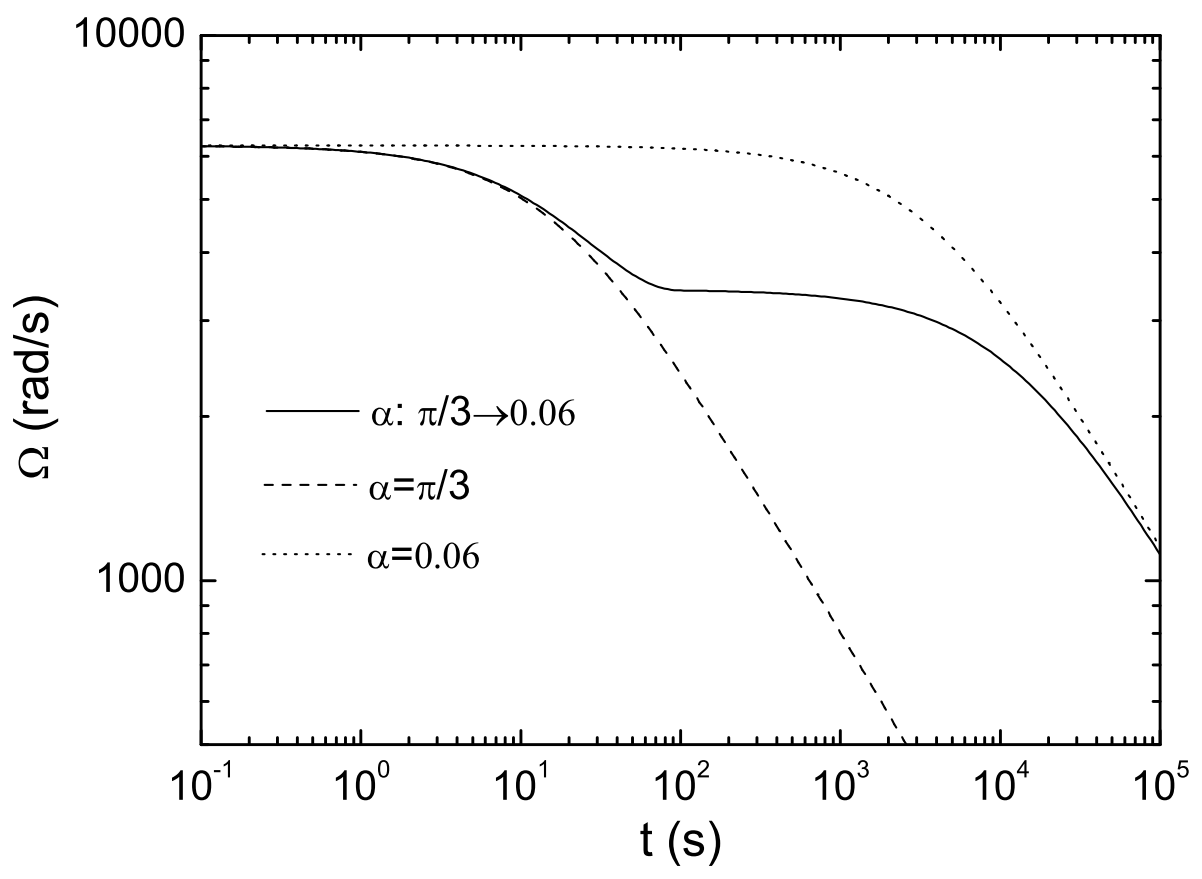

Figure 1: Evolution of the angular velocity of magnetars. The solid curve corresponds to our model with the tilt angle decreasing from $\pi / 3 \mathrm{rad}$ to $0.06 \mathrm{rad}$ within $100.0 \mathrm{~s}$. The dashed and dotted curves correspond to magnetars with fixed tilt angles of $\pi / 3 \mathrm{rad}$ and $0.06 \mathrm{rad}$, respectively [5].

the fixed scenario of $\pi / 3 \mathrm{rad}$. With the rapid decrease of the magnetic tilt angle, the magnetar then releases energy slower than the fixed tilt angle scenario, thus the evolution deviates from the dashed curve. One hundred seconds later, the tilt angle terminates at $\alpha_{\text {end }}=0.06 \mathrm{rad}$. Within this period, $\dot{E}$ evolves from the initial value of about $2.2 \times 10^{51} \mathrm{erg} / \mathrm{s}$ to about $9.0 \times 10^{47} \mathrm{erg} / \mathrm{s}$. Then, the evolution of $\dot{E}$ enters the second stage. At last, $\dot{E}$ decreases along the dotted curve.

Using the model above and estimated parameters in Table $\mathbb{U}$, we test the consistency of the model with the data of two GRB's light curves in Fig. B. The calculated curve of GRB 061121 is shown at the top of Fig. [1, and GRB 090426 at the bottom. The black points correspond to the light curve of X-ray afterglow observed by Swift $-X R T$. The dash lines correspond to the average $\gamma$-ray luminosity within $T_{90}$ of GRBs' prompt emission. The solid curves correspond to the calculated curves with our model. As is shown Fig. [3, the model's curves are well consistent with the data. The evolution of the energy loss rate consistent with the overall light curves of those gamma-ray bursts which show a plateau structure in the afterglow stage.

\section{Conclusion and Discussion}

Our study shows that the energy loss rate of a newborn magnetar with a rapidly decreasing magnetic tilt angle is very different from that of the fixed tilt angle scenario. Because of the decreasing magnetic tilt angle, the spin-down of the magnetar is characterized by two different 


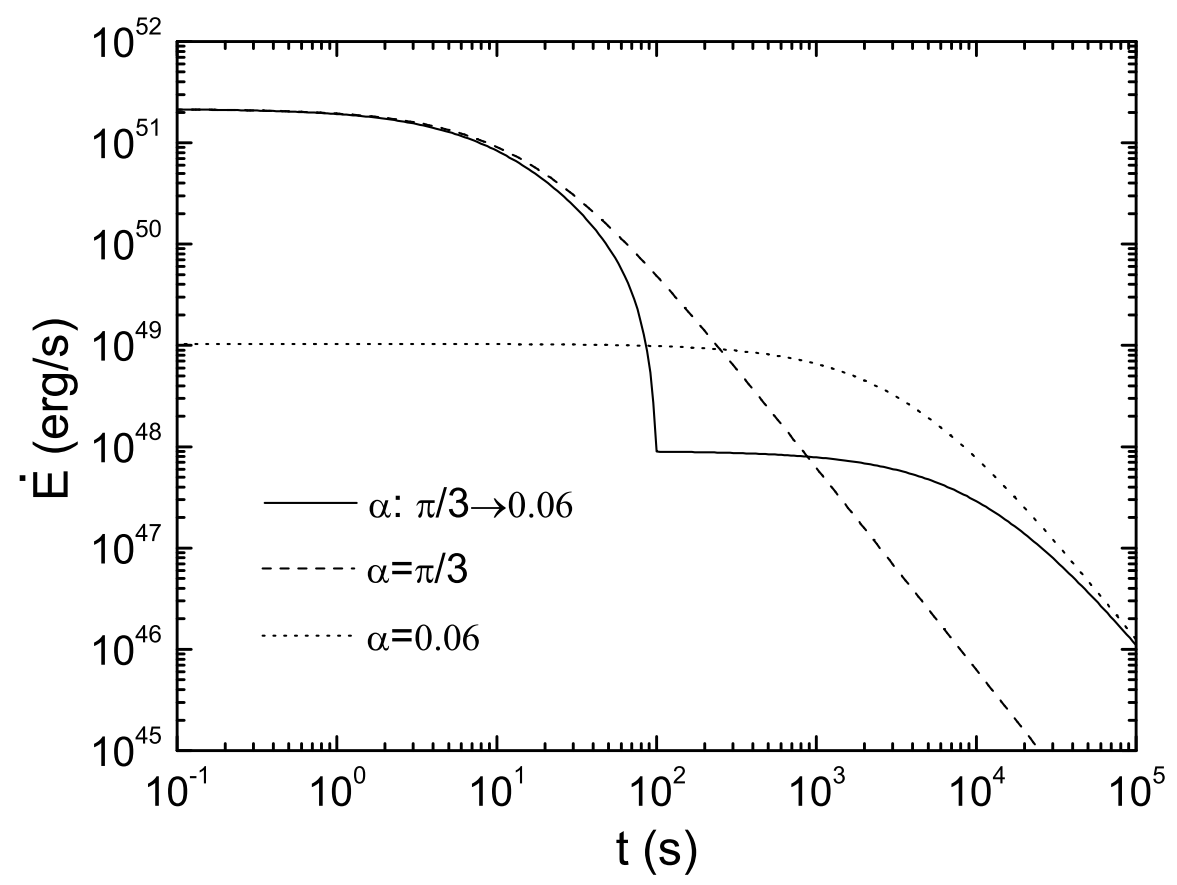

Figure 2: Evolution of the energy loss rate $(\dot{E})$ of magnetars during the spin-down. The solid curve corresponds to our model with a decreasing tilt angle. The dashed and dotted cures correspond to the evolution of $\dot{E}$ for fixed tilt angles of $\pi / 3$ and 0.06 rad, respectively [5].

\begin{tabular}{|lcccccc|}
\hline GRB & $\begin{array}{c}B_{\mathrm{p}} \\
{[\mathrm{G}]}\end{array}$ & $\begin{array}{c}P_{0} \\
{[\mathrm{sec}]}\end{array}$ & $\begin{array}{c}\alpha_{0} \\
{[\mathrm{rad}]}\end{array}$ & $\begin{array}{c}\alpha_{\text {end }} \\
{[\mathrm{rad}]}\end{array}$ & $\begin{array}{c}t_{\text {end }} \\
{[\mathrm{sec}]}\end{array}$ & $\begin{array}{c}I \\
{\left[\mathrm{~g} \cdot \mathrm{cm}^{2}\right]}\end{array}$ \\
\hline 061121 & $1.5 \mathrm{E} 16$ & $0.8 \mathrm{E}-3$ & 1.54 & 0.12 & 80 & $3.0 \mathrm{E} 45$ \\
090426 & $1.0 \mathrm{E} 17$ & $1.0 \mathrm{E}-3$ & 0.84 & 0.15 & 25 & $2.0 \mathrm{E} 45$ \\
\hline
\end{tabular}

Table 1: The parameters of GRB 061121 and GRB 090426 used in our model.

timescales. The energy loss rate duo to magnetic dipole radiation also experiences two stages. It changes from the initial value of $2.2 \times 10^{51} \mathrm{erg} / \mathrm{s}$ to about $9.0 \times 10^{47} \mathrm{erg} / \mathrm{s}$ within $100.0 \mathrm{~s}$ [可]. At first, the energy loss rate is large enough to power the GRB prompt emission. At the second stage, the energy loss rate can provide necessary energy injection for the GRB fireball and power the afterglow emission [焑].

Comparing the evolution curve of the energy loss rate with the overall GRB light curves (from the prompt emission to the afterglow stage), we find that they are very similar. So, we argue that some GRBs with a plateau phase in the afterglow stage may actually happen as follows. Initially, the newborn millisecond magnetar releases a huge amount of energy to launch a powerful ejecta to produce a burst. This corresponds to the prompt emission phase. As the tilt angle decreases rapidly, energy release from the magnetar also reduces markedly. Since the energy loss rate is no longer powerful enough to maintain the burst, a steep decay phase will naturally appear in the GRB light curve. When the tilt angle finally becomes stable, the energy loss rate enters a new stage. It 

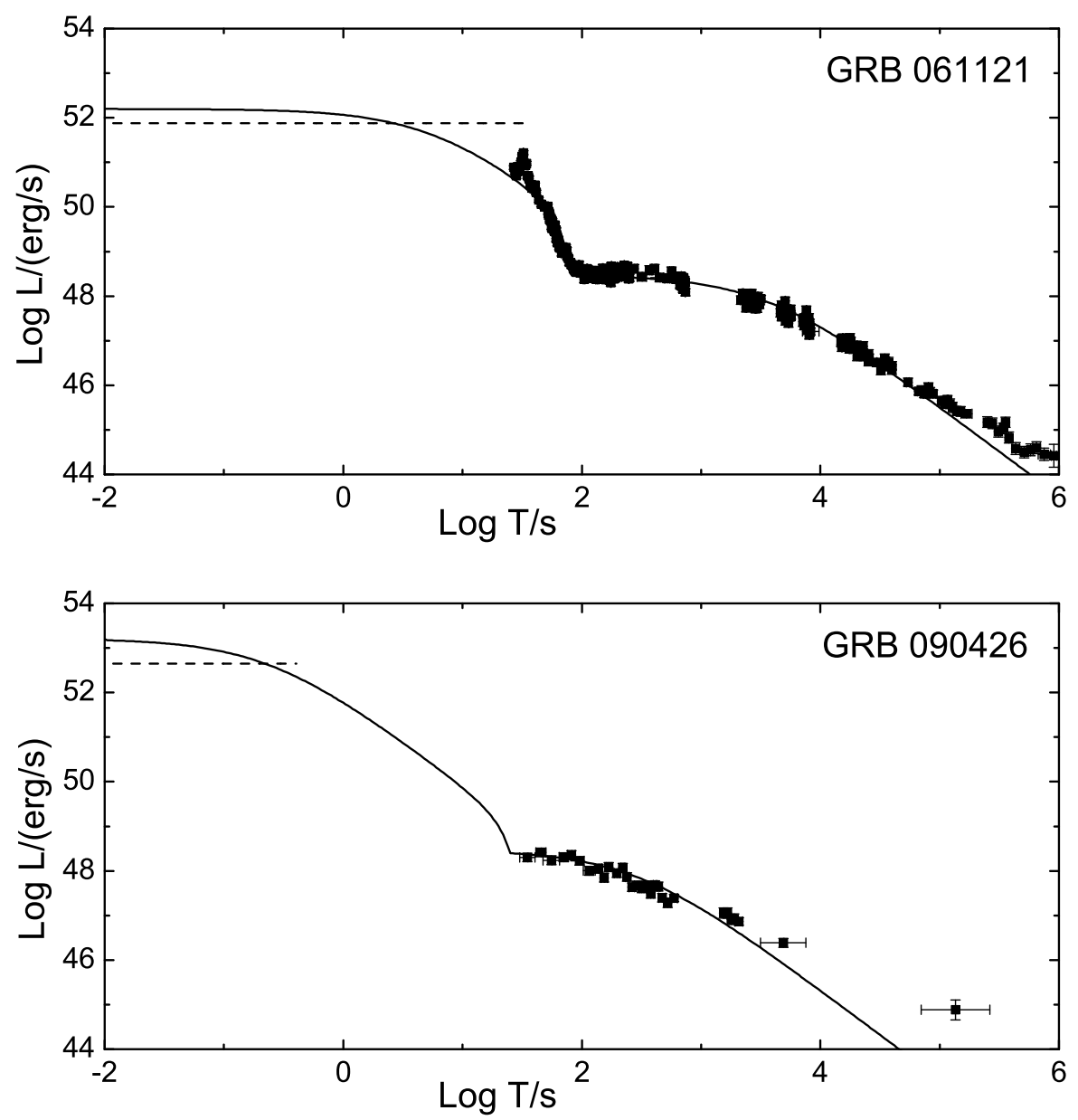

Figure 3: Testing our model with the data of light curves of GRB 061121 (top) and GRB 090426 (bottom). The black points correspond to the light curve of X-ray afterglow observed by Swift $-X R T$. The dash lines correspond to the average $\gamma$-ray luminosity within $T_{90}$ of GRBs' prompt emission. The solid curves correspond to the calculated curves with our model.

decreases slowly on a timescale of several thousand seconds and energy is continuously injected into the fireball. This stage corresponds to the shallow decay phase in the GRB afterglow light curve. At last, the energy injection comes to the end when most of the rotational energy of the magnetar is consumed. Then the afterglow enters the normal decay phase.

Therefore, the evolution of the energy loss rate in our model is consistent with the overall light curves of those gamma-ray bursts which show a plateau structure in the afterglow stage, such as GRB 061121 and GRB 090426 etc.

Our study supports the idea that those GRBs with a plateau structure in the afterglow light curve may be originated from newborn millisecond magnetars [प]. 


\section{References}

[1] V. Usov, Millisecond Pulsars with Extremely Strong Magnetic Fields as a Cosmological Source of Gamma-Ray Bursts, Nature 357 (1992) 472.

[2] Z. G. Dai, and T. Lu, Gamma-Ray Burst Afterglows and Evolution of Postburst Fireballs with Energy Injection from Strongly Magnetic Millisecond Pulsars, A\&A 333 (1998) L87 [astro-ph/9810402].

[3] M. Xu, Y. F. Huang and T. Lu, Long-term Continuous Energy Injection in the Afterglow of GRB 060729, RAA 9 (2009) 1317 [arXiv:0909.5318].

[4] S. Shapiro, and S. Teukolsky, Black Holes, White Dwarfs, and Neutron Stars: The Physics of Compact Objects, Wiley, New York 1983.

[5] M. Xu, and Y. F. Huang, Effect of Rapid Evolution of Magnetic Tilt Angle on Newborn Magnetar's Dipole Radiation, RAA (2015) accepted.

[6] N. Gehrels, E. Ramirez-Ruiz, and D. Fox, Gamma-Ray Bursts in the Swift Era, ARA\&A 47 (2009) 567 [arXiv:0909.1531].

[7] M. Xu, and Y. F. Huang, New three-Parameter Correlation for Gamma-Ray Bursts with a Plateau Phase in the Afterglow, A\&A 538 (2012) A134 [arXiv:1103.3978]. 